\title{
Insurance Risk with Variable Number of Policies
}

\author{
Ivo Adan* and Vidyadhar Kulkarni ${ }^{\dagger}$
}

April 27, 2007

\begin{abstract}
In this paper we consider an insurance company selling life insurance policies. New policies are sold at random points in time, and each policy stays active for a random amount of time, during which the policyholder pays premiums continuously at rate $r$. When the policy expires, the insurance company pays a claim of random size. The aim is to compute the probability of eventual ruin starting with a given number of policies and a given level of insurance reserves. We establish a remarkable result that, if the lifetimes of policies are i.i.d. exponential random variables with rate $\mu$, then the ruin probability is identical to the one in the standard compound Poisson model where the reserves increase at constant rate $r$ and claims occur according to a Poisson process with rate $\mu$. We conclude that the ruin probability does not depend on the initial number of active policies, nor on the arrival process of new policies.
\end{abstract}

\section{Introduction}

The most studied model in the literature on risk processes is the compound Poisson model. In this model, the insurance reserve increases at constant rate $r$ and claims arrive according to a Poisson process with rate $\mu$. The claim sizes are i.i.d. with common distribution $G$ and mean $\tau$, and are independent of the arrival process. Thus the reserve fund jumps down by random amounts at Poisson event times, and increases linearly between these events. Let $\psi_{P}(x)$ denote the probability that the insurance reserve eventually reaches a level of less than 0 , given that the initial fund is $x$. The probability $\psi_{P}(x)$ is called the infinite horizon ruin probability of the compound Poisson model. If $\tau \geq r / \mu$ the ruin probability is one, i.e., the ruin is certain. When $\tau<r / \mu$, it is well-known (see e.g. Rolski et al. [4], Theorem 5.3.3) that the ruin probability is less than one, and its Laplace transform

$$
\psi_{P}^{*}(s)=\int_{0}^{\infty} \psi_{P}(x) e^{-s x} d x, \quad s>0,
$$

*Department of Mathematics and Computer Science, Technische Universiteit Eindhoven, P.O. Box 513, 5600 MB, Eindhoven, The Netherlands. E-mail: iadan@win.tue.nl

$\dagger$ Department of Statistics and Operations Research, University of North Carolina, Chapel Hill, NC 27599. Email: vkulkarn@email.unc.edu 
is given by the Pollackzek-Khinchin formula

$$
\psi_{P}^{*}(s)=\frac{\rho}{s} \cdot \frac{1-\tilde{G}_{e}(s)}{1-\rho \tilde{G}_{e}(s)},
$$

where $\tilde{G}_{e}(s)$ is the Laplace-Stieltjes transform of the equilibrium distribution of $G$,

$$
\tilde{G}_{e}(s)=\int_{0}^{\infty} e^{-s x} \frac{1-G(x)}{\tau} d x, \quad s \geq 0
$$

and

$$
\rho=\frac{\mu \tau}{r}
$$

The motivation for the model studied in this paper comes from a more realistic model of life insurance companies. We assume that new life insurance policies are sold at random epochs and stay active for a random amount of time with mean $1 / \mu$. Let $X(t)$ be the number of policies active at time $t$. Initially, $X(0)$ policies are active, which were issued before or at time 0 . Note that $X=\{X(t), t \geq 0\}$ can be thought of as the number of customers in an infinite server queue. This justifies classifying the models using the queueing theoretic notation. We further assume that each active policy pays premiums continuously at constant rate $r$ to the insurance company. The instantaneous total rate at which premiums are paid to the company at time $t$ is thus equal to $r X(t)$. When a policy expires, the insurance company pays out a claim, the mean size of which is denoted by $\tau$. Let $\rho$ be as in Equation (2), and assume that

$$
\rho<1
$$

i.e., $r / \mu$, the expected premiums from a policy during its lifetime, is greater than $\tau$, the expected payout when the policy expires. The above condition is therefore the usual net profit condition.

Let $Z(t)$ be the insurance reserve level at time $t$. Thus $Z(t)$ is equal to the initial fund $Z(0)$ plus the total premiums collected over $[0, t]$ minus the total claims paid out over $[0, t]$. Let $T$ be the ruin time, defined by

$$
T=\inf \{t>0: Z(t)<0\} .
$$

The main quantity of interest is the infinite horizon ruin probability

$$
\psi(x, i)=P(T<\infty \mid Z(0)=x, X(0)=i), \quad x \geq 0, i=0,1, \ldots
$$

In this paper we compute the above probability. The complicating feature here is that the ruin function $\psi$ now depends on the bivariate process $(Z, X)=\{Z(t), X(t), t \geq 0\}$, and the remaining life-times of the $i$ policies in existence at time zero. Therefore, we start the analysis under the simplest possible assumptions, i.e., inter-arrival times, lifetimes and payouts are all exponential and independent of each other, and the number of 
active policies is bounded. From there on, we will explore the problem under more general circumstances. In doing so, we discover the remarkable result that, if the lifetimes of policies are exponential with rate $\mu$ and payouts are $i i d$ with distribution $G$, then $\psi(x, i)$ is the same as the ruin probability of the standard compound Poisson model where claims of size $G$ arrive according to a Poisson process with rate $\mu$, i.e., $\psi(x, i)=\psi_{P}(x)$ for all $i$. This implies that $\psi$ does not dependent on the initial number of active policies, nor on the arrival process of new policies.

Since the $X$ process can be thought of as a queue length process, we shall use the queueing theoretic notation for batch service queues to identify the insurance model under consideration. The symbol for the batch size distribution is taken to signify the claim size distribution.

\section{The $M / M^{M} / N / N$ and $M / M^{M} / \infty$ Models}

We begin with the $M / M^{M} / N / N$ model. Thus policies are sold according to a Poisson Process with rate $\lambda$. The lifetimes of new policies and the residual lifetimes of policies already active at time 0 are assumed to be iid exponentials with rate $\mu$, and independent of the arrival process. The payouts are iid exponentials with rate $\theta$ and independent of the arrivals and lifetimes. The company allows at most $N$ active policies. If it has $N$ active policies, any newly arriving policies are lost.

Let $X(t)$ be the number of policies that are active at time $t$. Then $X$ is a CTMC describing the number of customers in an $M / M / \infty$ queue with arrival rate $\lambda$ and service rate $\mu$.

One can think of the insurance reserve level process $Z$ as a Markovian fluid level modulated by $X$ as follows: while $X(t)=i, Z(t)$ increases at rate $i r$ and when $X$ jumps from $i$ to $i-1$, the $Z$ process instantaneously drops by an exponential amount. Sample paths of $X$ and $Z$ are shown in Figure 1.

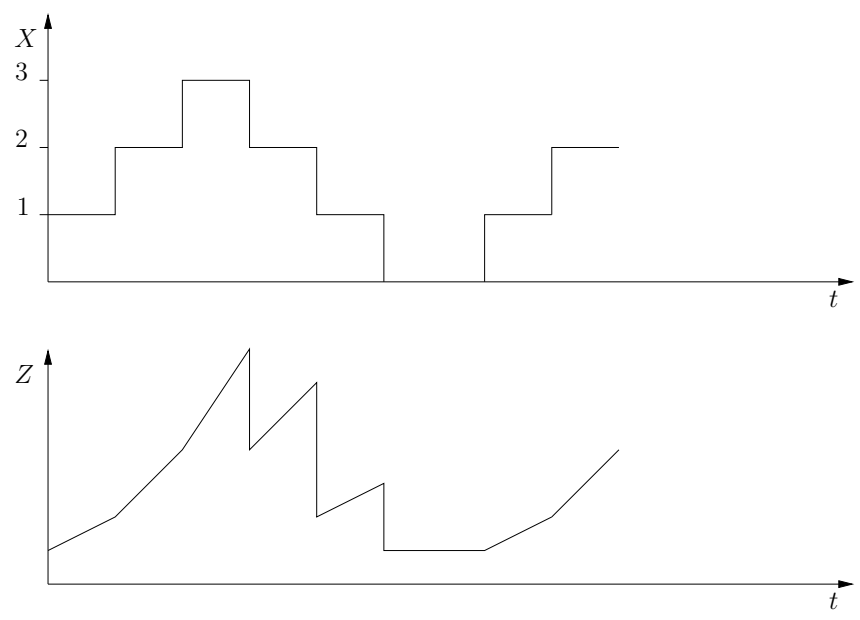

Figure 1: Sample paths of $X$ and $Z$ 
Next we create a new CTMC $Y=\{Y(t), t \geq 0\}$ and a new standard fluid level process $W=\{W(t), t \geq 0\}$ without any downward jumps. Sample paths of $Y$ and $W$ are shown in Figure 2.
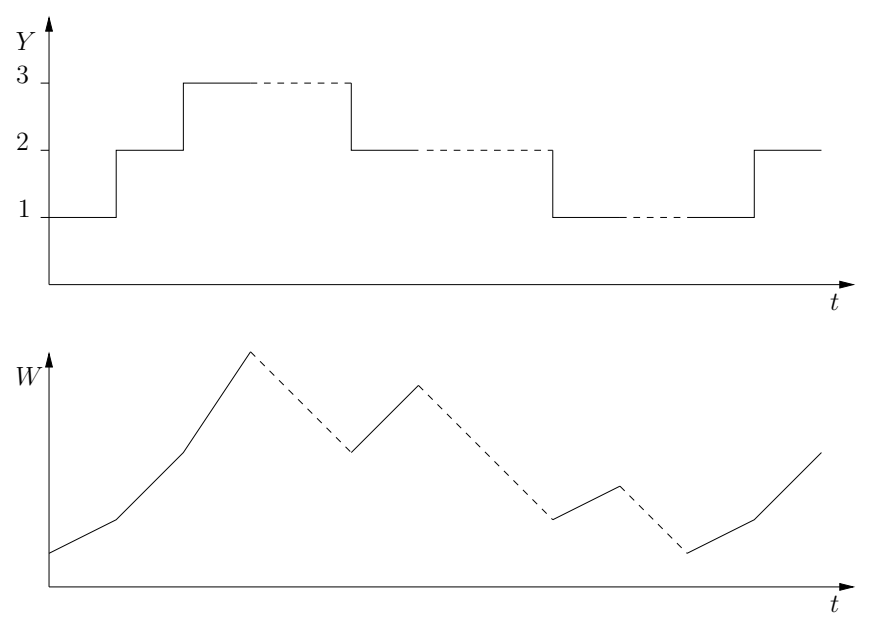

Figure 2: Sample paths of $Y$ and $W$

Intuitively, we get the sample path of the $W$ process by replacing every downward jump in the $Z$ process with a downward sloping line (with slope -1 ) creating an equal downward change, and by removing all segments where the $Z$ process is constant. When the $Z$ process is increasing at rate $i r$ we set $Y=(0, i)$, if the $Z$ process is decreasing at rate -1 , we set $Y=(1, i)$ if the $Z$ process was increasing at rate $i r$ just before it started decreasing. The solid lines in the sample path of $Y$ correspond to states of the type $(0, i)$, and the dashed lines in the sample path of $W$ are inserted sloping lines.

The state space of the $Y$ process is finite and given by $\{0,1\} \times\{1,2, \ldots, N\}$. The transition rates are

$$
\begin{aligned}
q_{(0, i),(0, i+1)} & \lambda, \quad 1 \leq i \leq N-1, \\
q_{(0, i),(1, i)} & =i \mu, \quad 1 \leq i \leq N, \\
q_{(1,1),(0,1)} & =\theta \\
q_{(1, i),(0, i-1)} & =\theta, \quad 2 \leq i \leq N .
\end{aligned}
$$

The fluid change rates of $W$ are

$$
\begin{aligned}
& r_{(0, i)}=i r, \quad 1 \leq i \leq N \\
& r_{(1, i)}=-1, \quad 1 \leq i \leq N .
\end{aligned}
$$

Thus while the $Y$ process is in state $(k, i)$, the $W$ process changes linearly at rate $r_{(k, i)}$. Now define

$$
U=\inf \{t>0: W(t)=0\}
$$


and let

$$
\hat{\psi}(x,(k, i))=P(U<\infty \mid W(0)=x, Y(0)=(0, i)), \quad k=0,1 ; 1 \leq i \leq N .
$$

Clearly, the transformation of $(X, Z)$ into $(Y, W)$ does not alter the ruin probability. Hence, we get

$$
\psi(x, i)=\hat{\psi}(x,(0, i)), \quad x \geq 0, i=1, \ldots
$$

We also have, $\psi(x, 0)=\psi(x, 1)=\hat{\psi}(x,(0,1))$ for all $x \geq 0$. Thus the problem of computing the ruin probability reduces to a first passage time problem in a standard fluid model. Hence, following the standard route (see Kulkarni and Tzenova [2]) we get the result in the following lemma. First, some notation: Let $Q$ be the $2 N \times 2 N$ generator matrix of $Y, R$ be a $2 N \times 2 N$ diagonal matrix $\operatorname{diag}\left[r_{(0,1)}, \cdots, r_{(0, N)}, r_{(1,1)}, \cdots, r_{(1, N)}\right]$, and $\hat{\psi}(x)$ be a $2 N \times 1$ column vector $[\hat{\psi}(x,(0,1)), \cdots, \hat{\psi}(x,(0, N)), \hat{\psi}(x,(1,1)), \cdots, \hat{\psi}(x,(1, N))]$.

Lemma 1. The ruin probability satisfies the system of differential equations

$$
R \frac{d}{d x} \hat{\psi}(x)+Q \hat{\psi}(x)=0, \quad x>0
$$

with boundary conditions

$$
\hat{\psi}(0,(1, i))=1, \quad i=1,2, \ldots, N .
$$

Proof. For $x \geq 0,0<i<N$ and small $h>0$ we have

$$
\begin{aligned}
\hat{\psi}(x,(0, i))= & (1-(\lambda+i \mu) h) \hat{\psi}(x+i r h,(0, i))+\lambda h \hat{\psi}(x+i r h,(0, i+1)) \\
& +i \mu h \hat{\psi}(x+i r h,(0, i-1))+o(h) .
\end{aligned}
$$

Hence,

$$
\begin{aligned}
-i r \frac{\hat{\psi}(x+i r h,(0, i))-\hat{\psi}(x,(0, i))}{i r h}= & -(\lambda+i \mu) \hat{\psi}(x+i r h,(0, i))+\lambda \hat{\psi}(x+i r h,(0, i+1)) \\
& +i \mu \hat{\psi}(x+i r h,(0, i-1))+o(1) .
\end{aligned}
$$

Letting $h \rightarrow 0$ yields the desired equation. The equations for states $(1, i)$ can be obtained similarly assuming $x>0$. The boundary condition is obvious.

Together with the boundary conditions $\hat{\psi}(0,(1, i))=1$ for all $1 \leq i \leq N$ and $\hat{\psi}(x) \rightarrow 0$ as $x \rightarrow \infty$, the above system of differential equations has a unique solution, which can be determined by the usual spectral techniques (see Kulkarni [3]). Typically, the solution is given in terms of $N$ exponentials, but surprisingly, a single exponential suffices for the present problem, as given in the following theorem.

Theorem 1. The ruin probability $\psi(x, i)$ is given by

$$
\psi(x, i)=\rho \exp (-\theta(1-\rho) x)
$$

for all $x \geq 0$ and $i=0,1, \ldots, N$. 
Proof. By writing Equation (7) in scalar form we get

$$
\begin{aligned}
i r \frac{d}{d x} \hat{\psi}(x,(0, i))-(\lambda+i \mu) \hat{\psi}(x,(0, i))+\lambda \hat{\psi}(x,(0, i+1)) & \\
+i \mu \frac{d}{d x} \hat{\psi}(x,(1, i)) & =0, \quad 1 \leq i<N, \\
N r \frac{d}{d x} \hat{\psi}(x, N)-N \mu \hat{\psi}(x,(0, N))+N \mu \hat{\psi}(x,(1, N)) & =0, \\
-\frac{d}{d x} \hat{\psi}(x,(1,1))-\theta \hat{\psi}(x,(1,1))+\theta \hat{\psi}(x,(0,1)) & =0, \\
-\frac{d}{d x} \hat{\psi}(x,(1, i))-\theta \hat{\psi}(x,(1, i)) & \\
+\theta \hat{\psi}(x,(0, i-1)) & =0, \quad 2 \leq i \leq N .
\end{aligned}
$$

To solve the above system of differential equations we take as trial solution,

$$
\hat{\psi}(x,(k, i))=\phi_{0}(x), \quad 1 \leq i \leq N, k=0,1 .
$$

Substitution of the trial solution into the system of differential equations yields

$$
\begin{aligned}
r \frac{d}{d x} \phi_{0}(x)-\mu \phi_{0}(x)+\mu \phi_{1}(x) & =0, \\
-\frac{d}{d x} \phi_{1}(x)-\theta \phi_{1}(x)+\theta \phi_{0}(x) & =0 .
\end{aligned}
$$

These equations can be solved in closed form to get

$$
\begin{aligned}
& \phi_{0}(x)=\rho \exp (-\theta(1-\rho) x), \\
& \phi_{1}(x)=\exp (-\theta(1-\rho) x) .
\end{aligned}
$$

Hence, the required ruin probability is given by $\phi_{0}(x)$ for all initial number of policies. Hence, using Equation (6), we get Equation (9) for $1 \leq i \leq N$. Finally we note that $\psi(x, 0)=\psi(x, 1)$, hence Equation (9) holds for $i=0$ as well.

The result in Theorem 1 is remarkable: the ruin function $\psi(x, i)$ is independent of $i$ and is also independent of $\lambda>0$ ! Hence, we can immediately conclude the following result for the $M / M^{M} / \infty$ model.

Corollary 1. In case $N=\infty$, the ruin probability $\psi(x, i)$ is also given by (9) for all $x \geq 0$ and $i=0,1, \ldots$

\section{The $M / M^{P H} / N / N$ and $M / M^{P H} / \infty$ Models}

In this section we consider the $M / M^{P H} / N / N$ model. The main assumptions are the same as in the $M / M^{M} / N / N$ model of the previous section, except that the claim sizes are $i$ id phase type random variables with parameters $(\alpha, M)$. Here $M=\left[m_{i, j}\right]_{i, j=1,2, \ldots, K}$ is an invertible (or transient) generator matrix with non-positive row sums of a CTMC 
on the state space $\{1,2, \ldots, K\}$, and $\alpha=\left[\alpha_{1}, \alpha_{2}, \ldots, \alpha_{K}\right]$ is a probability vector; $\alpha_{k}$ is the probability that the phase type random variable starts in state $k$.

As before, we transform $(X, Z)$ into a standard fluid model $(Y, W)$. However, when the $W$ process is decreasing, the $Y$ process keeps track of the current state of the phase type distribution. The state space of $Y$ is now $\{0,1, \cdots, K\} \times\{1,2, \cdots, N\}$ with transition rates given below:

$$
\begin{aligned}
q_{(0, i),(0, i+1)} & =\lambda, \quad 1 \leq i \leq N-1 \\
q_{(0, i),(k, i)} & =i \mu \alpha_{k}, \quad 1 \leq i \leq N, 1 \leq k \leq K \\
q_{(k, 1),(0,1)} & =\theta \sum_{j=1}^{K} M_{k j}, \quad 1 \leq k \leq K \\
q_{(k, i),(0, i-1)} & =\theta \sum_{j=1}^{K} M_{k j}, \quad 2 \leq i \leq N, 1 \leq k \leq K .
\end{aligned}
$$

When the $Y$ process is in state $(0, i)$, the $W$ process increases at rate $i r$, when it is in state $(k, i)$, the $W$ process changes at rate -1 . Let $U$ be as defined in Equation (4) and extend the definition of $\hat{\psi}$ of Equation (5) to $k=0,1, \ldots, K$. Clearly Equation (6) remains valid, and the problem reduces to a first passage problem for the new $(Y, W)$ process. Now, let $Q$ be the $(K+1) N \times(K+1) N$ generator matrix of $Y, R$ be a $(K+1) N \times(K+1) N$ diagonal matrix $\operatorname{diag}\left[r_{(0,1)}, \cdots, r_{(0, N)}, r_{(1,1)}, \cdots, r_{(1, N)}, \cdots, r_{(K, 1)}, \cdots, r_{(K, N)}\right]$, and $\hat{\psi}(x)$ be a $(K+$ 1) $N \times 1$ column vector $[\hat{\psi}(x,(0,1)), \cdots, \hat{\psi}(x,(0, N)), \cdots, \hat{\psi}(x,(K, 1)), \cdots, \hat{\psi}(x,(K, N))]$. Lemma 1 remains valid with the modified boundary conditions:

$$
\hat{\psi}(0,(k, i))=1, \quad 1 \leq i \leq N, 1 \leq k \leq K .
$$

To solve the system of differential equations (7), we take as trial solution,

$$
\hat{\psi}(x,(k, i))=\phi_{k}(x), \quad 1 \leq i \leq N, 0 \leq k \leq K .
$$

Let $\phi(x)=\left[\phi_{1}(x), \ldots, \phi_{k}(x)\right]^{T}$, where the superscript $T$ represents the transpose. Substitution of the trial solution into the system of differential equations yields

$$
\begin{aligned}
r \frac{d}{d x} \phi_{0}(x)-\mu \phi_{0}(x)+\mu \alpha \phi(x) & =0 \\
-\frac{d}{d x} \phi(x)+M \phi(x)-M e \phi_{0}(x) & =0 .
\end{aligned}
$$

where $e$ is the column vector of all ones. The boundary conditions are $\phi(0)=e$ and $\phi_{0}(x), \phi(x) \rightarrow 0$ as $x \rightarrow \infty$. Taking the Laplace transforms

$$
\phi_{0}^{*}(s)=\int_{0}^{\infty} e^{-s x} \phi_{0}(x) d x, \quad \phi^{*}(s)=\int_{0}^{\infty} e^{-s x} \phi(x) d x, \quad s>0,
$$

yields

$$
\begin{aligned}
-r \phi_{0}(0)+r s \phi_{0}^{*}-\mu \phi_{0}^{*}+\mu \alpha \phi^{*} & =0 \\
e-s \phi^{*}+M \phi^{*}-M e \phi_{0}^{*} & =0
\end{aligned}
$$


The transform $\phi_{0}^{*}$ can be solved from the above equations to obtain

$$
\phi_{0}^{*}=\frac{r \phi_{0}(0)-\mu \alpha(s I-M)^{-1} e}{r s-\mu\left(1+\alpha(s I-M)^{-1} M e\right)} .
$$

Note that

$$
1+\alpha(s I-M)^{-1} M e=\alpha\left(I+(s I-M)^{-1} M\right) e=\alpha s(s I-M)^{-1} e,
$$

so Equation (13) can be simplified to

$$
\phi_{0}^{*}=\frac{r \phi_{0}(0)-\mu \alpha(s I-M)^{-1} e}{s\left(r-\mu \alpha(s I-M)^{-1} e\right)} .
$$

It remains to determine $\phi_{0}(0)$. The boundary condition $\phi_{0}(x) \rightarrow 0$ as $x \rightarrow \infty$ implies that $s \phi_{0}^{*}(s) \rightarrow 0$ as $s \rightarrow 0$. Thus, from (14) we get

$$
\lim _{s \rightarrow 0} s \phi_{0}^{*}(s)=\frac{r \phi_{0}(0)+\mu \alpha M^{-1} e}{r+\mu \alpha M^{-1} e}=\frac{r \phi_{0}(0)-\mu \tau}{r-\mu \tau},
$$

where we used that $\tau=-\alpha M^{-1} e$. Hence, we can conclude that $\phi_{0}(0)=\rho$. Thus the solution given in Equation (12) is valid. It is independent of $i$ and of $\lambda$ and $N$ ! Thus we get the following theorem for the $M / M^{P H} / \infty$ model.

Theorem 2. In the case of $N=\infty$, the Laplace transform of the ruin probability $\psi(x, i)$ is given by

$$
\psi^{*}(s, i)=\int_{0}^{\infty} e^{-s x} \psi(x, i) d x=\frac{\rho}{s} \cdot \frac{1-(\alpha / \tau)(s I-M)^{-1} e}{1-\rho(\alpha / \tau)(s I-M)^{-1} e}
$$

for all $s>0$ and $i=0,1, \ldots$

Now, if the claim size $C$ is phase-type distributed with parameters $(\alpha, M)$, the LaplaceStieltjes transform of the equilibrium distribution of the claim size is given by

$$
\tilde{C}_{e}(s)=\int_{0}^{\infty} e^{-s x} \frac{1-P(C \leq x)}{\tau} d x=\frac{1}{\tau} \alpha(s I-M)^{-1} e .
$$

Hence, Theorem 2 can be rewritten as:

Theorem 3. The Laplace transform of the ruin probability $\psi(x, i)$ is given by

$$
\psi^{*}(s, i)=\frac{\rho}{s} \cdot \frac{1-\tilde{C}_{e}(s)}{1-\rho \tilde{C}_{e}(s)}, \quad s>0, i=0,1, \ldots
$$

This immediately suggests a generalization to general claim size distributions. We study this generalization in the next section. 


\section{The $M / M^{G} / N / N$ and $M / M^{G} / \infty$ Models}

In this section we consider the generalization of the models in the previous section by assuming that the claim sizes are iid general non-negative random variables with common cdf $G$. Let $G_{e}$ be the corresponding equilibrium distribution. We shall prove the following theorem.

Theorem 4. The Laplace transform of the ruin probability $\psi(x, i)$ in the $M / M^{G} / \infty$ model is given by

$$
\psi^{*}(s, i)=\frac{\rho}{s} \cdot \frac{1-\tilde{G}_{e}(s)}{1-\rho \tilde{G}_{e}(s)}, \quad s>0, i=0,1, \ldots
$$

To prove the above theorem, we begin with the $M / M^{G} / N / N$ model. The following lemma states the integral-differential equation satisfied by $\psi(x, i)$.

Lemma 2. The ruin probabilities satisfy the system of integral-differential equations

$$
\begin{aligned}
& \operatorname{ir} \frac{d}{d x} \psi(x, i)-(\lambda+i \mu) \psi(x, i)+\lambda \psi(x, i+1) \\
& \quad+i \mu(1-G(x))+i \mu \int_{0}^{x} \psi(x-u, i-1) d G(u)=0, \quad 1 \leq i<N, \\
& N r \frac{d}{d x} \psi(x, N)-N \mu \psi(x, N) \\
& \quad+N \mu\left(1-G(x)+N \mu \int_{0}^{x} \psi(x-u, N-1) d G(u)=0,\right.
\end{aligned}
$$

and $\psi(x, 0)=\psi(x, 1)$.

Proof. For $x \geq 0,1 \leq i<N$ and small $h>0$ we have

$$
\begin{aligned}
\psi(x, i)= & (1-(\lambda+i \mu) h) \psi(x+i r h, i)+\lambda h \psi(x+i r h, i+1) \\
& +i \mu h(1-G(x+i r h))+i \mu h \int_{0}^{x+i r h} \psi(x+i r h-u, i-1) d G(u)+o(h) .
\end{aligned}
$$

Rearranging the terms we get

$$
\begin{aligned}
-i r \frac{\psi(x+i r h, i)-\psi(x, i)}{i r h}= & -(\lambda+i \mu) \psi(x+i r h, i)+\lambda \psi(x+i r h, i+1) \\
& +i \mu(1-G(x+i r h)) \\
& +i \mu \int_{0}^{x+i r h} \psi(x+i r h-u, i-1) d G(u)+o(1) .
\end{aligned}
$$

Letting $h \rightarrow 0$ yields the desired equation; the equation for $i=N$ can be obtained similarly. Further, $\psi(x, 0)=\psi(x, 1)$, since the fund level stays constant while $i=0$. 
Together with the boundary conditions $\psi(x, i) \rightarrow 0$ as $x \rightarrow \infty$, the above system of integral-differential equations has a unique solution. To solve this system we take as trial solution,

$$
\psi(x, i)=\phi(x), \quad 0 \leq i \leq N .
$$

Substitution of the trial solution into the system of integral-differential equations yields

$$
r \frac{d}{d x} \phi(x)-\mu \phi(x)+\mu(1-G(x))+\mu \int_{0}^{x} \phi(x-u) d G(u)=0 .
$$

Taking the Laplace transform

$$
\phi^{*}(s)=\int_{0}^{\infty} e^{-s x} \phi(x) d x, \quad s>0,
$$

we obtain

$$
r\left(-\phi(0)+s \phi^{*}\right)-\mu \phi^{*}+\mu \tau \tilde{G}_{e}+\mu \phi^{*} \tilde{G}=0 .
$$

Simplifying, we get

$$
\phi^{*}=\frac{r \phi(0)-\mu \tau \tilde{G}_{e}}{r s-\mu+\mu \tilde{G}}=\frac{1}{s} \cdot \frac{\phi(0)-\rho \tilde{G}_{e}}{1-\rho(1-\tilde{G}) /(\tau s)} .
$$

Since

$$
\tilde{G}_{e}=\frac{1-\tilde{G}}{\tau s}
$$

expression (16) reduces to

$$
\phi^{*}=\frac{1}{s} \cdot \frac{\phi(0)-\rho \tilde{G}_{e}}{1-\rho \tilde{G}_{e}} .
$$

Finally, the unknown $\phi(0)$ follows from the condition that $s \phi^{*}(s) \rightarrow 0$ as $s \rightarrow 0$. Since

$$
\lim _{s \rightarrow 0} s \phi^{*}(s)=\frac{\phi(0)-\rho}{1-\rho},
$$

we conclude that $\phi(0)=\rho$, and hence

$$
\psi^{*}(s, i)=\phi^{*}(s)=\frac{\rho}{s} \cdot \frac{1-\tilde{G}_{e}(s)}{1-\rho \tilde{G}_{e}(s)}, \quad s>0,0 \leq i \leq N .
$$

Clearly, $\psi^{*}(s, i)$ does not depend on $i$, which implies that Equation (17) is also valid for the case of $N=\infty$. This completes the proof of Theorem 4 .

Interestingly enough, the result in Equation (15) is the same formula as in the standard ruin problem, where the premiums come in at a constant rate $r$, and claims arise according to a Poisson process with rate $\mu$, and the claim sizes are iid with common distribution $G$; see (1). In our model, the average rate at which premiums come in is $(\lambda / \mu) r$ and the average rate at which claims arise is $(\lambda / \mu) \mu$. This might be an intuitive explanation for the independence of our result on $\lambda$. 
The absence of the arrival rate $\lambda$ in the formula for the ruin probability suggests that the result may be valid for any arrival process. Further, it is still not clear why the initial number of active policies does not affect the ruin probability. In the next section we study the model with general arrivals, and show, essentially by a sample path argument, that the result in Theorem 4 remains valid under these circumstances.

\section{The $G / M^{G} / \infty$ Model}

In this section we study the $G / M^{G} / \infty$ model, i.e., the new policies arrive according to an arbitrary arrival process. All other assumptions are the same as in the previous section: the lifetimes of policies are exponential with rate $\mu$ and the claims are iid with common distribution $G$. We assume that, initially $i$ policies are active; these policies were issued before or at time 0 . Let $S_{j}$ be the time of sale (issue) of the $j$-th new policy and let $C_{j}$ be the size of the $j$-th claim. The sequences $\left\{S_{j}\right\}$ and $\left\{C_{j}\right\}$ are independent of each other. The assumption of exponential lifetimes will appear to be critical in the analysis.

Now, throughout the remainder of this section, we consider a fixed realization of the sequence of sales epochs $0<S_{1}<S_{2}<\cdots$. In Figure 3 we display a realization of the fund level process $Z$, where $E_{j}$ denotes the $j$-th inter-event time. The realization of $Z$ is the same as the one in Figure 1. Below we will show that the fund level process $Z$ can be transformed into the fund level process of the standard compound Poisson model, yielding the same ruin probability. The transformation is first explained intuitively.

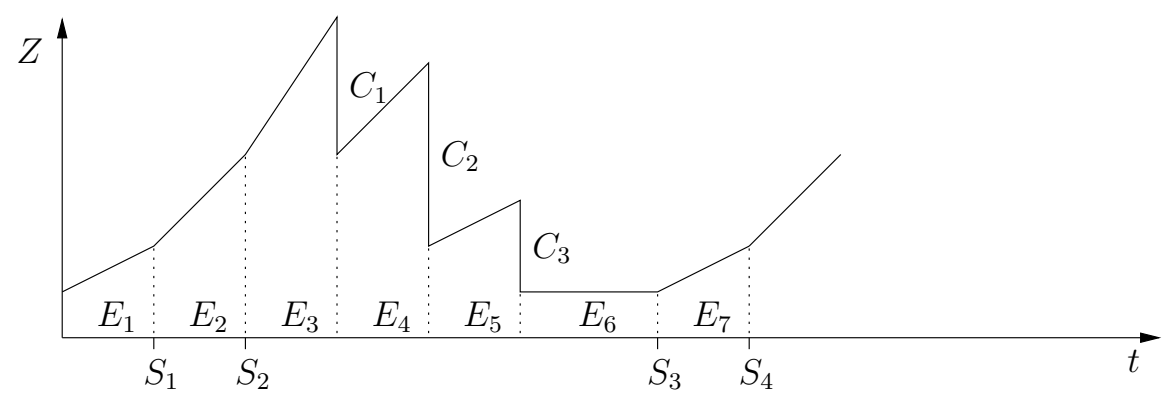

Figure 3: Sample path of the fund level process $Z$ with, initially, 1 active policy

At time 0 the fund increases with rate $i r$, and after an exponential time with rate $i \mu$, one of the policies will expire. The idea is to make the clock tick $i$ times slower until the first event, i.e., an arrival of a new policy or an active policy expires. To put it differently, we stretch the time axis (make it $i$ times longer) until the first event. The result is that the fund will increase at rate $r$ and, after an exponential time with rate $\mu$, one of the $i$ active policies will expire; the first arrival is now rescheduled at time $S_{1}^{\prime}=i S_{1}$. Of course, slowing down time does not affect the ruin probability.

In case a policy expires before the arrival scheduled at time $S_{1}^{\prime}$, say at time $D$, the fund level decreases by an amount $C_{1}$, and after this, $i-1$ active policies remain. So we adapt 
the clock again and now make it run $i-1$ times slower, so the fund continues to increase with rate $r$ and after an exponential time with rate $\mu$ one of the $i-1$ remaining policies will expire; the arrival is now rescheduled at $S_{1}^{\prime}=D+(i-1)\left(i S_{1}-D\right) / i$. If zero policies would remain, then we simply remove the time period up to the first arrival at $S_{1}^{\prime}$ (the fund remains constant over this period).

In the other case, a new policy arrives at time $S_{1}^{\prime}$. Then we adapt the clock again and make it run $i+1$ times slower, so that immediately after the arrival, the fund will continue to increase at the same rate $r$, and after an exponential time with rate $\mu$ one of the $i+1$ active policies will expire. The next arrival is scheduled at time $S_{2}^{\prime}=S_{1}^{\prime}+(i+1)\left(S_{2}-S_{1}\right)$.

Note that, in the construction outlined above, we use the memoryless property of exponentials, i.e., after an event the remaining lifetimes of the active policies are again exponential with the same rate $\mu$. The construction of dynamically adjusting the speed of time is illustrated in Figure 4 for the sample shown in Figure 3. Note that the interevent time $E_{6}$ has been deleted, since the fund level stays constant during the period of length $E_{6}$.

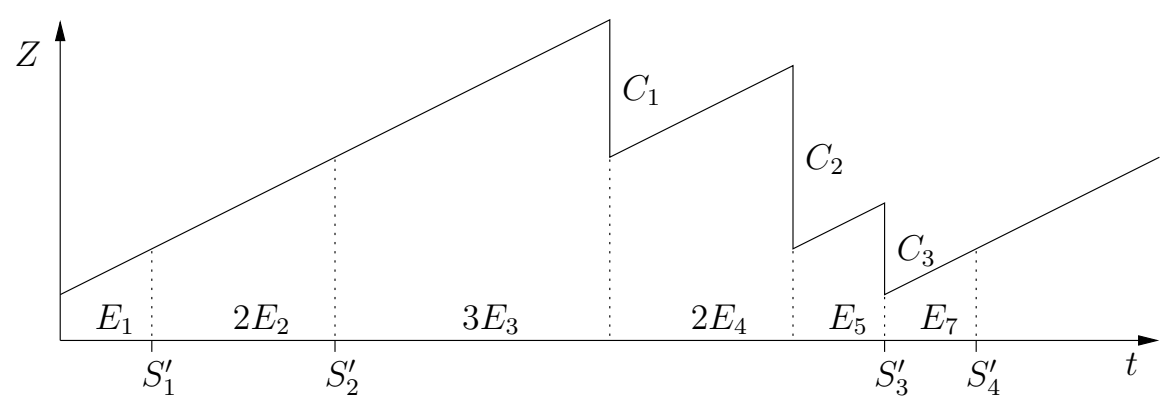

Figure 4: The construction of dynamically adjusting the speed of time applied to the sample path in Figure 3

The result of this construction is that the fund level process does not notice any changes in the number of active policies! This explains why the arrival process and the initial number are not relevant to the ruin probability. Also, the time in between subsequent claims is always exponentially distributed with rate $\mu$.

By formalizing the above transformation of time we prove the following theorem.

Theorem 5. For a fixed realization of the sequence of sales epochs, the ruin probability $\psi(x, i)$ is exactly the same as the ruin probability $\psi_{P}(x)$ for the compound Poisson model, the Laplace transform of which is given by (1).

To prove Theorem 5 we first need to define the time transformation formally. We map each time point $t$ to a new point $\tau(t)$ defined by

$$
\tau(t)=\int_{0}^{t} X(u) d u .
$$


Clearly, all time points in an interval during which $X$ is zero are mapped to the same point. Now define the (generalized) inverse mapping of $\tau$ as

$$
\sigma(t)=\sup \left\{s: \int_{0}^{s} X(u) d u=t\right\}
$$

So, if $\tau$ maps multiple time points to the same point, the inverse of $\tau$ takes the right most time point (for which $X$ is equal to 1 ). The mapping $\tau$ transforms $(Z, X)$ into $(Z(\sigma), X(\sigma))$. We first establish the following lemma.

Lemma 3. The transformed fund level process $Z(\sigma)$ is stochastically identical to the fund level process in the standard compound Poisson model.

Proof. Although the original fund level process $Z$ is regulated by $X$, we will show that the transformed process $Z(\sigma)$ no longer depends on $X(\sigma)$. The amount $Z(\sigma)$ increases in the interval $[t, t+d t]$, given that $X(\sigma(t))=i$, is equal to

$$
i r \frac{d t}{i}=r d t
$$

and the probability that a claim occurs in $[t, t+d t]$, given that $X(\sigma(t))=i$, is equal to

$$
\mu i \frac{d t}{i}=\mu d t
$$

Hence, the rate at which the fund level process $Z(\sigma)$ increases is always $r$ and the rate at which a claim occurs is always $\mu$, independent of $X(\sigma)$. In other words, $Z(\sigma)$ is stochastically identical to the fund level process in the standard compound Poisson model, where the fund increases at constant rate $r$ and claims arrive according to a Poisson stream with rate $\mu$, the sizes of which are iid with distribution $G$. This proves the lemma.

We now return to the proof of the theorem. Since the time transformation $\tau$, which dynamically slows down the speed of time and removes time periods during which the fund level stays constant, has no effect on the (infinite horizon) ruin probability, we can immediately conclude that the ruin probability in the original model is exactly the same as in the standard compound Poisson model. This completes the proof of Theorem 5.

Clearly, the ruin probability $\psi(x, i)$ is the same for every realization of the sales process. Hence, we immediately obtain:

Corollary 2. For an arbitrary arrival process of new policies, the ruin probability $\psi(x, i)$ is exactly the same as the ruin probability $\psi_{P}(x)$ for the compound Poisson model, the Laplace transform of which is given by (1).

The assumption of iid exponential lifetimes is critical to Theorem 5: the result is no longer true for non-exponential lifetimes, nor for the finite horizon ruin probability. Note, however, that the method based on the construction of a standard Markovian fluid model, as illustrated in Section 2 and 3, can be applied to compute the finite horizon ruin probability. 


\section{Exponential Approximation}

In this section we present a simple approximation of the ruin probability $\psi(x, i)$ for exponential lifetimes and general claim sizes; as we have seen in the previous section, $\psi(x, i)$ is equal to $\psi_{P}(x)$.

Using Equation (1) we get

$$
\begin{aligned}
\psi_{P}(0) & =\rho \\
\int_{0}^{\infty} \psi_{P}(x) d x & =\frac{\rho}{1-\rho} \frac{s^{2}}{2 \tau}
\end{aligned}
$$

where $s^{2}$ is the second moment of the claim size. This implies that if we want to approximate $\psi_{P}(x)$ by an exponential function

$$
\psi_{P}(x)=a \exp (-b x)
$$

satisfying the two equations above, we are forced to choose

$$
a=\rho
$$

and

$$
b=\frac{2(1-\rho) \tau}{s^{2}} .
$$

Note that the approximation is exact when the payout distribution is exponential. Also, this is identical to the exponential approximation to the waiting time in an $M / G / 1$ queue, see [1]

Below we include some numerical results demonstrating the quality of the proposed approximation. We compute the ruin probabilities for the following distributions of the claim sizes: Exponential(1), Erlang(5,.2), Uniform over $(0,2)$, Constant, and Hyperexponential-4 with mixing probability vector $[.1, .2, .3, .4]$ and corresponding mean vector $[2,1.5,1,2]$. The mean claim size in each case is $\tau=1$; the second moment $s^{2}$ is equal to $2,1.2,1.33,1$ and 2.7 in these five cases. We take $\mu=.9$, and $r=1$, so that $\rho=\mu \tau / r=.9$ for all the five cases. For each case we also compute the exponential approximation. The results are plotted in the Figure 5 for the initial reserve level $x$ ranging from 0 to 40. The lowest graph belongs to the constant, the next higher one to Erlang case, the next one to the uniform case, the next one to the exponentials case, and topmost one to the Hyperexponential case. In effect, the graphs are arranged from bottom to top in increasing value of the second moment. It is clear from Figure 5 that the exponential approximation works very well.

The relative error (exact ruin probability minus the exponential approximation) in using the exponential approximation in place of exact calculation is plotted in Figure 6. Clearly the approximation is exact in the exponential case. The lowest graph corresponds to the Hyperexponential case, the next one to the Erlang case, the next one to the constant case and the topmost one to the uniform case. 


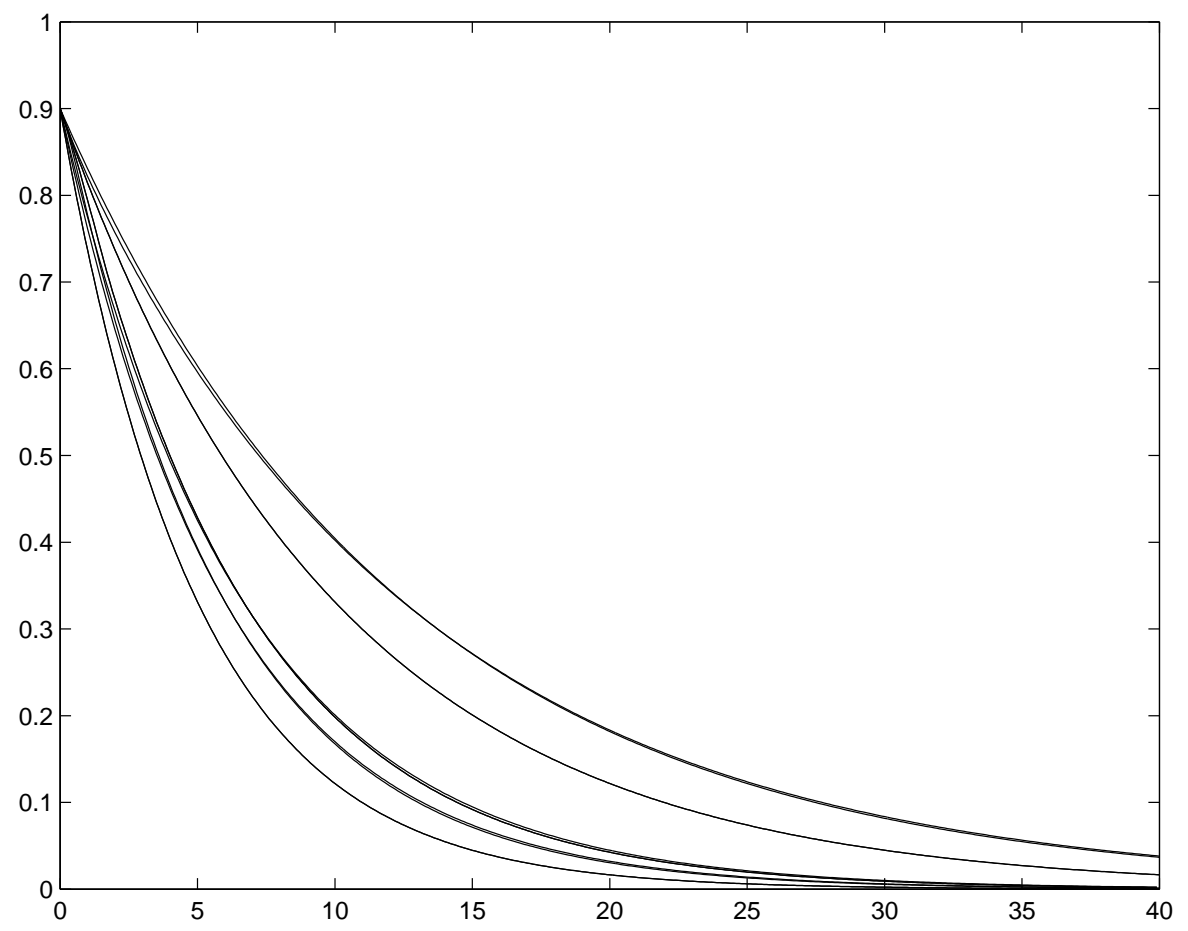

Figure 5: Ruin probabilities and their approximation for different claim size distributions

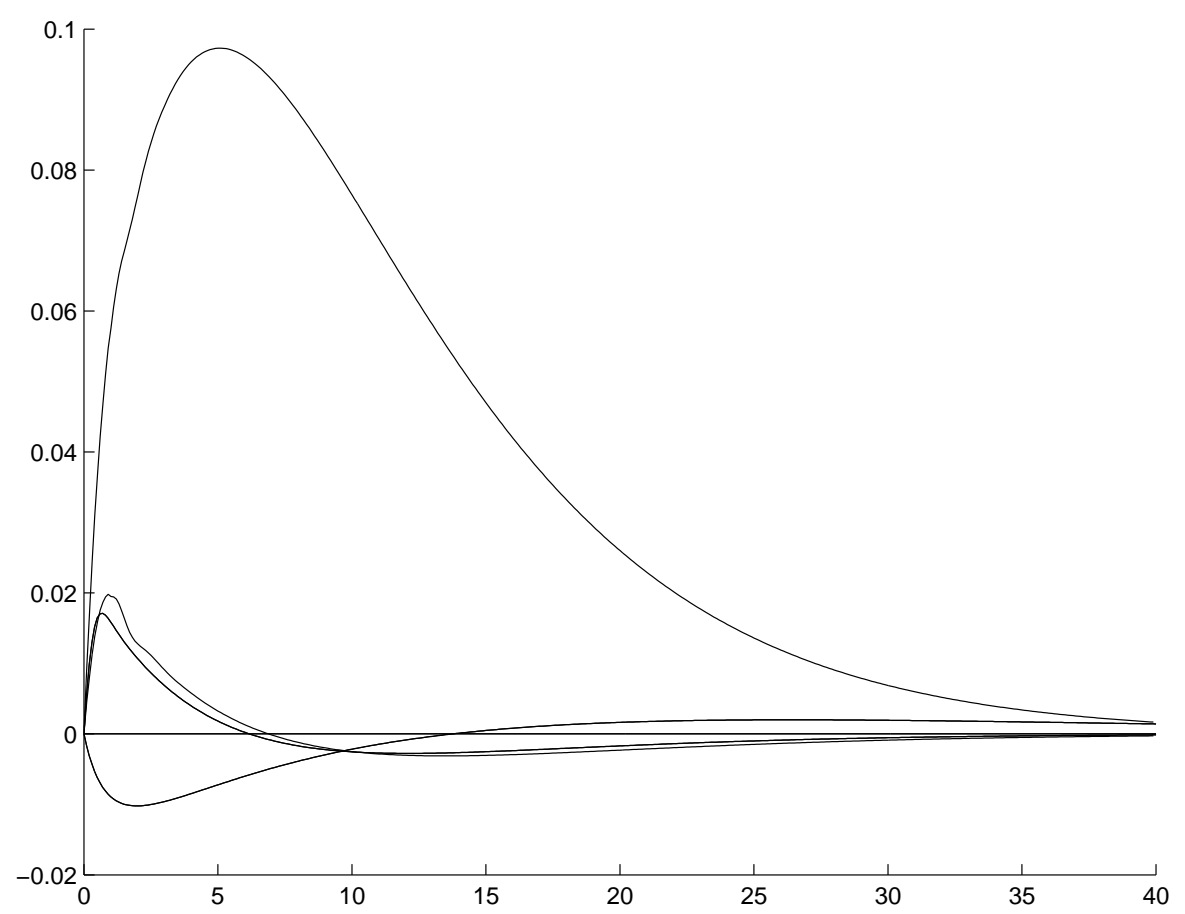

Figure 6: Relative error of the exponential approximation for different claim size distributions 


\section{Conclusions}

In this paper we have studied a problem faced by a company that sells life insurance policies. The sales occur according to a general process, the lifetimes of the individual policies are iid exponential $(\mu)$ random variables during which each policy-holder pays premiums at a common fixed rate $r$, the pay-outs at the expiry of the policies are iid random variables with common distribution $G$. We assume the net profit condition and compute the infinite horizon ruin probability starting with a given reserve level and a given number of policies. We have shown two surprising results: (1) the ruin probability is independent of the arrival process and the initial number of policies, and (2) the ruin probability is the same as in the standard model where the premiums accumulate a fixed rate $r$, and the claims occur according to a Poisson process with rate $\mu$, and the claim sizes are iid with common distribution $G$. These results are valid for any arbitrary sales process!

The crucial assumption is the exponential distribution of lifetimes. The results are no longer true for non-exponential lifetimes, nor for the finite horizon ruin probability. It should be noted, however, that the construction of standard Markovian fluid models, as presented in Section 2 and 3, can also be applied to compute the finite horizon ruin probability.

\section{References}

[1] Heyman, D. and M. Sobel. (1984). Stochastic Models in Operations Research, Vol I. McGraw Hill, NY.

[2] Kulkarni, V. G. and E. Tzenova (2002). Mean First Passage Times in Fluid Queues, OR Letters, 30, 308-318.

[3] Kulkarni, V. G. (1997). Fluid models for single buffer systems, Frontiers in Queueing: Models and Applications in Science and Engineering, 321-338, Ed. J. H. Dshalalow, CRC Press.

[4] Rolski, T., H. Schmidli, V. Schmidt and J. Teugels (1999). Stochastic processes for insurance and finance. John Wiley 8 Sons, Chichester. 